\title{
DESIGN, DEVELOPMENT, CHARACTERIZATION AND COMPARISON OF CALCIUM SILICATE AND CHOLESTYRAMINE BASED ELETRIPTAN HYDROBROMIDE FLOATING MICROSPHERES
}

\author{
Saleha, K. Shravan Kumar, 'D. Prabhakar, U. Ashok kumar \\ Department of Pharmaceutics, Trinity College of Pharmaceutical Sciences, Peddapalli, Karimnagar- 505172, Andhra Pradesh, India. \\ *Corresponding Author's Email: shravankonda@yahoo.com
}

\begin{abstract}
:
Studies were carried out to develop, characterize and compare oral floating microspheres for Eletriptan Hydrobromide using Ion exchange resin like Cholestyramine and low density porous carrier like calcium silicate so as to provide extended gastric retention. The prepared resinates were studied for the effect of $\mathrm{pH}$ and drug resin ratio on drug loading and is similar with calcium silicate. Both the formulations were evaluated for drug entrapment, in vitro floating behavior, in vivo buoyancy and in vitro drug release studies. Dissolution study of Eletriptan hydrobromide microspheres with both the calcium silicate and cholestyramine was able to sustain the release of the drug. The study suggests that the floating microspheres of Eletriptan hydrobromide provide sustained release over $24 \mathrm{~h}$ and also showed in vivo buoyancy for more than $10 \mathrm{~h}$ in rabbit.
\end{abstract}

Keywords:

\section{INTRODUCTION:}

The word new or novel in the relation to drug delivery system is a search for something out of necessity. An appropriately designed sustained or controlled release drug delivery system can be major advance toward solving the problem associated with the existing drug delivery system

1,2 . Various attempts have been made to prolong the gastric residence time of dosage form in the stomach. Floating drug delivery system have a bulk density less than gastric fluids and so remains buoyant in the stomach without affecting gastric emptying rate for a prolonged period of time. While the system is floating on the gastric contents, the drug is released slowly at the desired rate from the system. After release of drug, the residual system is emptied from stomach.

This result in an increased gastric residence time and a better control of the fluctuation in plasma drug concentration ${ }^{3}$. Single unit formulations are associated with problems such as sticking together or being obstructed in the gastrointestinal tract, which may have a potential danger of producing irritation. On the other hand a floating system made of multiple unit forms has relative merits compared to a single unit preparation. Indeed the gastric emptying of a microparticulate floating system would occur in consistent manner with small individual variations on each subsequent gastric emptying sunken particles will spread out over a large area of absorption sites, increasing the opportunity of drug release and absorption in a more or less predictable way. Moreover, since each dose consists of many subunits, the risk of dose duping is reduced ${ }^{4}$. Floating microspheres provide a constant and prolonged therapeutic effect which will reduce dosing frequency. ${ }^{5,6}$ Eletriptan Hydrobromide is an antimigraine drug; its mechanism of action is explained by two theories to explain the efficacy of 5-HT receptor agonists in migraine. One theory the suggests that activation of 5-HT1 receptors located on intracranial blood vessels, including those on the arterio venous anastomoses,
leads to vasoconstriction, which is correlated with the $^{2}$ relief of migraine headache. The other hypothesis suggests that activation of 5-HT1 receptors on sensory nerve endings in the trigeminal system results in the inhibition of proinflammatory neuropeptide release. Inhibition of prostaglandin synthetase and its plasma elimination half life is $4 \mathrm{hrs}$ and in order to maintain therapeutic plasma level drug must be administered at least twice a day ${ }^{7}$.

The aim of this study was to prepare Eletriptan Hydrobromide floating microspheres containing hydrophobic polymer ethyl cellulose along with low density calcium silicate porous carrier and ion exchange resin Cholestyramine to achieve better encapsulation efficiency, better buoyancy and sustained drug release profile suitable for oral administration. The influences of formulation variables on the microsphere properties were examined and the microsphere formulations suitable to achieve our goal were determined.

\section{MATERIALS AND METHOD:}

\subsection{Materials:}

Eletriptan Hydrobromide was received as a gift sample from Finosa Pharmaceuticals, Hyderabad, India. Calcium silicate was procured from Johnson \& George Chemical company, Mumbai, Ethyl cellulose was a gift sample from Merck, Mumbai, Acetonitrile, Dichloromethane and Light liquid paraffin and other chemicals were of analytical grade.

\subsection{Methods:}

\subsubsection{Preparation of Eletriptan Hydrobromide} adsorbed Calcium silicate:

The porous carrier was dispersed into $10 \mathrm{ml}$ water solution of drug (Eletriptan Hydrobromide). This solution was ultrasonicated to imbibe the drug solution inside the pores of porous carrier ${ }^{8}$.

Preparation of Eletriptan Hydrobromide Cholestyramine complex: 
An accurately weighed amount of Eletriptan Hydrobromide $(100 \mathrm{mg}$ ) was taken and added in $10 \mathrm{ml}$ of distilled water. Then known weight of ion exchange resin was added to the solution and stirred on propeller stirrer. Resinate thus formed was washed with $0.1 \mathrm{~N} \mathrm{HCl}$ to remove the unloaded drug. Resinate was dried overnight in an hot air oven at $50^{\circ} \mathrm{C} 9$.

\subsubsection{Preparation of floating microspheres:}

Microspheres were prepared using a modified water-in oilin oil $\left(\mathrm{W} / \mathrm{O}_{1} / \mathrm{O}_{2}\right)$ double emulsion solvent diffusion method $^{10}$. Briefly, for calcium silicate and cholestyramine based microspheres, the drug adsorbed calcium silicate and drug- cholestyramine complex was added respectively to the polymer solution of ethyl cellulose (EC) in acetonitrile and dichloromethane (CAN:DCM) (1:1) and stirred using a mechanical stirrer at $500 \mathrm{rpm}$. The resulting $\mathrm{W} / \mathrm{O}$ primary emulsion was slowly added to $50 \mathrm{ml}$ of light liquid paraffin, the second oil phase containing $0.5 \%$ span 80 as a surfactant while stirring by a paddle propeller at $1000 \mathrm{rpm}$. After $2 \mathrm{~h}, 10 \mathrm{ml}$ of $n$-hexane (non-solvent) was added to harden the microspheres and stirring was continued for a further $1 \mathrm{~h}$ and the hardened microspheres were collected by filtration and washed thrice with $50 \mathrm{ml}$ of $n$-hexane and air dried for $12 \mathrm{~h}$. The effect of process variables like polymer concentration, stirring rate, temperature, concentration of aqueous phase on the particle size, buoyancy, drug entrapment efficiency, drug release, amount of porous carrier and ion exchange resin were studied.

Process variables: Amount of Polymer: 1000, 1500 mg; Amount of carrier: 150, 200, and 250.

\section{Characterization of microspheres:}

\subsection{Micromeritic properties:}

The microspheres were characterized by their micromeritic properties, such as particle size, true density, tapped density, compressibility index and flow properties. The size was measured using an optical microscope, and the mean particle size was calculated by measuring 200-300 particles with the help of a calibrated ocular micrometer. The tapping method was used to determine the tapped density and percent compressibility index ${ }^{11}$ as follows:

Tapped density = Mass of microspheres / Volume of microspheres after tapping

$$
\% \text { Compressibility index }=(1-\text { V/Vo }) \times 100
$$

Here $\mathrm{V}$ and Vo are the volumes of the sample after and before the standard tapping, respectively.

True density was determined using a benzene displacement method.

Porosity $(\varepsilon)$ was calculated using the equation:

$$
\varepsilon=\left(1_{-} \mathrm{Pp} / \mathrm{Pt}\right) \times 100
$$

where $\mathrm{Pt}$ and $\mathrm{Pp}$ are the true density and tapped density, respectively.

Angle of repose $\mathrm{h}$ of the microspheres, which measures the resistance to particle flow, was determined by a fixed funnel method. ${ }^{12}$ and calculated as $\operatorname{Tan} \Theta=2 \mathrm{~h} / \mathrm{D}$
Where $2 \mathrm{~h} / \mathrm{D}$ is the surface area of the free standing height of the microspheres heap that is formed on a graph paper after making the microspheres flow from the glass funnel.

\subsection{Morphology:}

The external and internal morphology of the microparticles and porous carrier were studied by scanning electron microscopy (SEM). The samples for SEM were pre-pared by lightly sprinkling the powder on a double adhesive tape stuck to an aluminum stub. The stubs were then coated with gold to a thickness of about $300 \mathrm{~A}^{\circ}$ under an argon atmosphere using a gold sputter module in a high-vacuum evaporator. The coated samples were then randomly scanned and photomicrographs were taken with a scanning electron microscope (Jeol JSM-1600, Tokyo, Japan).

\subsection{Determination of drug content of microspheres:}

Drug amount in microspheres was determined by dissolving $10 \mathrm{mg}$ of each sample in $100 \mathrm{ml}$ dichloromethane. The drug concentration was determined spectrophotometrically (UV-160, Shimadzu, Japan) at $272.2 \mathrm{~nm}$. All experiments were done in triplicate ${ }^{9}$.

\subsection{Determination of production yield:}

The production yield of the microparticles was determined by calculating accurately the initial weight of the raw materials and the last weight of the microspheres obtained $^{13}$. All of the experiments were performed in triplicate

$\%$ drug entrapment $=$ [calculated drug concentration/theoretical drug concentration] $\times 100$.

$\%$ yield $=$ [total weight of floating microparticles $/$ total weight of drug, polymer, porous carrier/resin (if added) $\times 100$.

\subsection{In -vivo floating behavior:}

Microspheres $(50 \mathrm{mg}$ ) were spread over the surface of a USP XXIV dissolution apparatus type II filled with $900 \mathrm{ml}$ of $0.1 \mathrm{~N}$ Hydrochloric acid. The medium was agitated with a paddle rotating at $100 \mathrm{rpm}$ for $12 \mathrm{~h}$. The floating and the settled portions of microspheres were recovered separately. The microspheres were dried and weighed. Buoyancy percentage was calculated as the ratio of the mass of the microspheres that remained floating and the total mass of the microspheres ${ }^{14}$.

$$
\text { Buoyancy }=\mathrm{Wf} /(\mathrm{Wf}+\mathrm{Ws}) \times 100
$$

Where Wf and Ws are the weights of the floating and settled microparticles, respectively. All the determinations were made in triplicate.

\subsection{In- vitro release studies:}

The drug release rate from floating microspheres was determined using USP XXIII basket type dissolution apparatus. A weighed amount of floating microspheres equivalent to $20 \mathrm{mg}$ drug was filled into a capsule and placed in the basket. $0.1 \mathrm{~N} \mathrm{HCl}$ was used as the dissolution medium and maintained at $37 \pm 0.5^{\circ} \mathrm{C}$. at a rotation speed of $100 \mathrm{rpm}$. Perfect sink conditions prevailed during the drug release studies. Five $\mathrm{ml}$ sample was withdrawn at each $30 \mathrm{~min}$ interval, passed through a membrane filter (Millipore), and analyzed spectrophotometrically at 272.2 


\section{Shravan et al}

Journal of Drug Delivery

$\mathrm{nm}$ to determine the concentration of drug present in the dissolution medium. The initial volume of the dissolution fluid was maintained by adding $5 \mathrm{ml}$ of fresh dissolution fluid after each withdrawal. All experiments were conducted in triplicate.

\subsection{In- vivo floating behaviour:}

Barium sulphate loaded microspheres were prepared by adopting the procedure as described before except for using barium sulphate instead of drug. Healthy rabbit weighing approximately $2.3 \mathrm{Kg}$ was used to assess in vivo floating behaviour. Ethical clearance for the handling of experimental animals was obtained from the SLS/2012/10/5/2(1047/ac/07/CPCSEA), constituted for the purpose. The animal was fasted for $12 \mathrm{~h}$ and the first $\mathrm{X}$-ray photographed to ensure absence of radio opaque material in the stomach. The rabbit were made to swallow barium sulphate loaded microspheres with $30 \mathrm{ml}$ of water. During the experiment rabbit were not allowed to eat but water was provided. At predetermined time intervals the radiograph of abdomen was taken using an X-ray machine ${ }^{15}$.

\subsection{Accelerated stability studies:}

Drug decomposition or degradation occurs during storage, because of chemical alteration of the active ingredients or due to product instability, leading to lower concentration
106

of the drug in the dosage form, hence the stability of pharmaceutical preparation need to be evaluated. The objective of stability studies is to predict the shelf life of a product by accelerating the rate of decomposition, preferably by increasing the temperature and relative humidity $(\mathrm{RH})$ conditions. Stability studies were carried out at $40 \pm 2{ }^{\circ} \mathrm{C}$ and $75 \pm 5 \%$ relative humidity for 90 days ${ }^{16}$.

\section{RESULTS AND DISCUSSIONS:}

\subsection{Formation of microspheres:}

Microspheres were formed after a series of steps like solvent extraction and solvent evaporation and addition of non-solvent. Acetonitrile is a unique organic solvent which is polar, water miscible and oil immiscible. All other polar solvents are oil-miscible and do not form emulsions of the polymer solution in oil ${ }^{17}$. Dichloromethane is non-polar and oil miscible. Using acetonitrile alone as a solvent did not ensure formation of a stable emulsion, and non-polar solvent such as dichloromethane was included to decrease polarity of the acetonitrile solution ${ }^{18}$. Therefore, during the formation of microspheres, dichloromethane is extracted by liquid paraffin and acetonitrile is evaporated during stirring.The incorporation of drug adsorbed calcium silicate or drug - cholestyramine complex into the formulation may produce porous structure within the microspheres. The compositions are showed in Table.1.

Table 1: Composition of floating microspheres of Eletriptan hydrobromide

EC: Ethylcellulose, (ACN: DCM): Acetonitrile: Dichloromethane

\begin{tabular}{|c|c|c|c|c|c|}
\hline Formulation & Drug (mg) & $\mathbf{E C}(\mathbf{m g})$ & Calcium silicate (mg) & $\begin{array}{c}\text { Cholestyra-mine } \\
(\mathbf{m g})\end{array}$ & ACN:DCM(1:1)(ml) \\
\hline CS1A & 100 & 1000 & 150 & - & 20 \\
\hline CS1B & 100 & 1000 & 200 & - & 20 \\
\hline CS1C & 100 & 1000 & 250 & - & 20 \\
\hline CS2A & 100 & 1500 & 150 & - & 20 \\
\hline CS2B & 100 & 1500 & 200 & - & 20 \\
\hline CS2C & 100 & 1500 & 250 & 150 & 20 \\
\hline CH1A & 100 & 1000 & - & 200 & 20 \\
\hline CH1B & 100 & 1000 & - & 250 & 20 \\
\hline CH1C & 100 & 1000 & - & 150 & 20 \\
\hline CH2A & 100 & 1500 & - & 200 & 20 \\
\hline CH2B & 100 & 1500 & - & 250 & 20 \\
\hline CH2C & 100 & 1500 & - & & \\
\hline
\end{tabular}

\subsection{Micromeritic properties:}

The mean particle sizes of calcium silicate powder was $142 \pm 02 \mu \mathrm{m}$, while that of microsphere formulations containing calcium silicate in the range of 150 to $250 \mathrm{mg}$ measured $650 \pm 12 \mu \mathrm{m}$ to $828 \pm 12 \mu \mathrm{m}$ when compared to cholestyramine measured $605 \pm 15 \mu \mathrm{m}$ to $813 \pm 14 \mu \mathrm{m}$. the particle size of microsphere formulation without buoyant material was found to be $180 \pm 08 \mu \mathrm{m}$. The tapped density values of calcium silicate ranged from $0.33 \pm 0.04 \mathrm{~g} / \mathrm{cm}^{3}$ to $0.65 \pm 0.06 \mathrm{~g} / \mathrm{cm}^{3}$ that of tapped density values of cholestyramine ranged from $0.43 \pm 0.09 \mathrm{~g} / \mathrm{cm}^{3}$ to $0.68 \pm 0.07 \mathrm{~g} / \mathrm{cm}^{3}$, while true density of calcium silicate ranged between $1.66 \pm 0.12 \mathrm{~g} / \mathrm{cm}^{3}$ and $1.84 \pm 0.1 \mathrm{~g} / \mathrm{cm}^{3}$ and cholestyramine ranged between $1.89 \pm 0.15 \mathrm{~g} / \mathrm{cm}^{3}$ and
$2.11 \pm 0.5 \mathrm{~g} / \mathrm{cm}^{3}$. The significant difference in the densities may be caused by the presence of low density calcium silicate and cholestyramine particles in the microspheres. The porosity of all the microsphere formulations was found to be in the range of $70.6 \% \pm 2.5 \%$ to $90.0 \% \pm 4.0 \%$. The compressibility index of calcium silicate based formulations ranged between $14.73 \% \pm 2.2 \%$ and $24.6 \% \pm 3.1 \%$ while that of calcium silicate based formulations ranged between $15.12 \% \pm 4.2 \%$ and $28.71 \% \pm 4.3 \%$. All formulations of calcium silicate showed excellent flow ability as expressed in terms of angle of repose $\left(<40^{\circ}\right)$ except formulation $\mathrm{CS} 2 \mathrm{C}$, probably due to higher content of calcium silicate, while in cholestyramine based formulations expressed flow ability in terms of angle of repose $\left(<46^{\circ}\right)$ except formulation $\mathrm{CH} 2 \mathrm{C}$, probably due 
to higher content of cholestyramine. The better flow property indicates that the floating microspheres produced are non-aggregated.

\subsection{Morphology:}

Calcium silicate and cholestyramine based Ethyl cellulose microspheres were predominantly spherical in appearance; however some were found to be elongated. The porous nature and spherical shape of the microspheres are evident from their SEM photomicrographs Fig. 1, Fig. 2. As can be seen in the photomicrograph, there are many pores and cavities in the microspheres. Low-density drug adsorbed FLR particle is clearly visible inside the microsphere in Fig. 3 and Fig. 4, which make them float on the simulated GIT fluids shows the surface morphology of microsphere at a higher magnification.

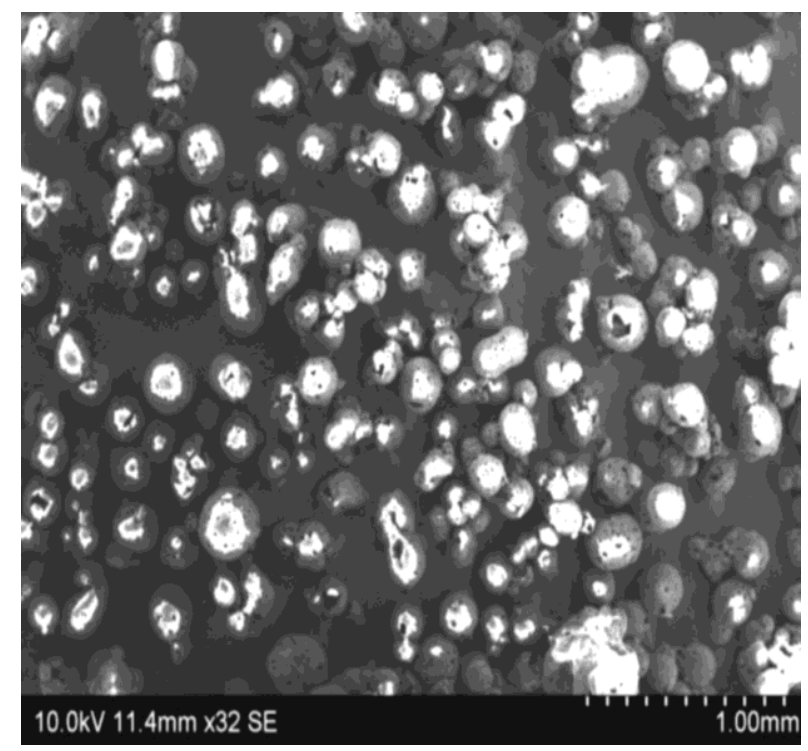

Fig. 1: SEM photomicrographs showing porous nature and spherical shape of calcium silicate based microspheres.

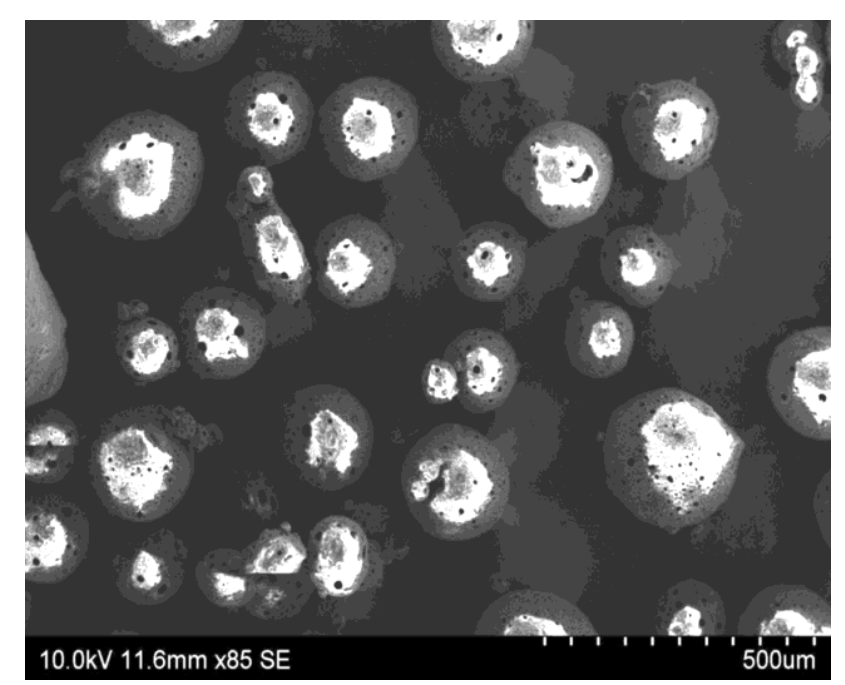

Fig. 2: SEM photomicrograph depicting many pores and cavities in the cholestyramine based microspheres.

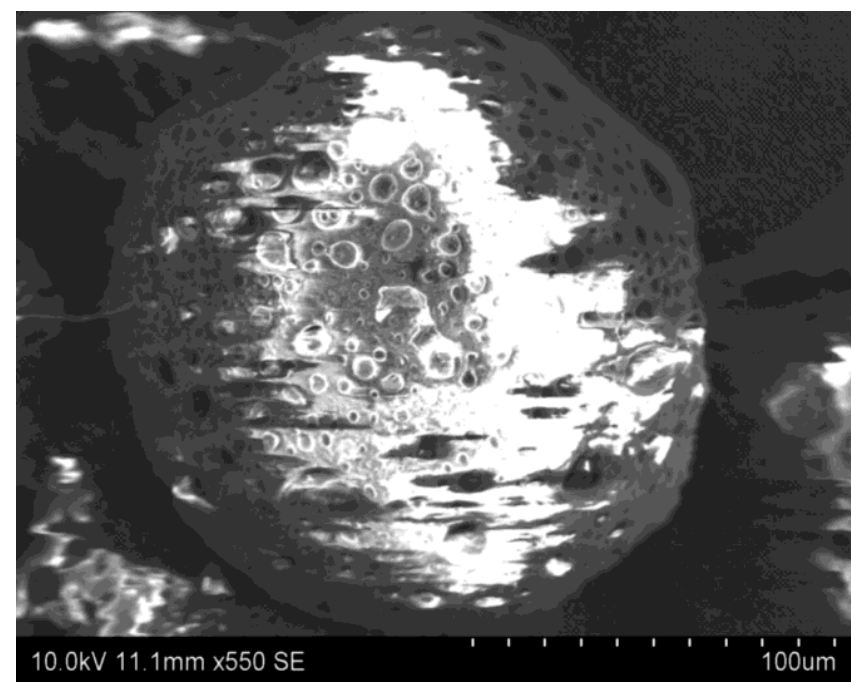

Fig. 3: SEM photomicrograph showing low-density drug adsorbed calcium silicate particle is clearly visible inside the microsphere.

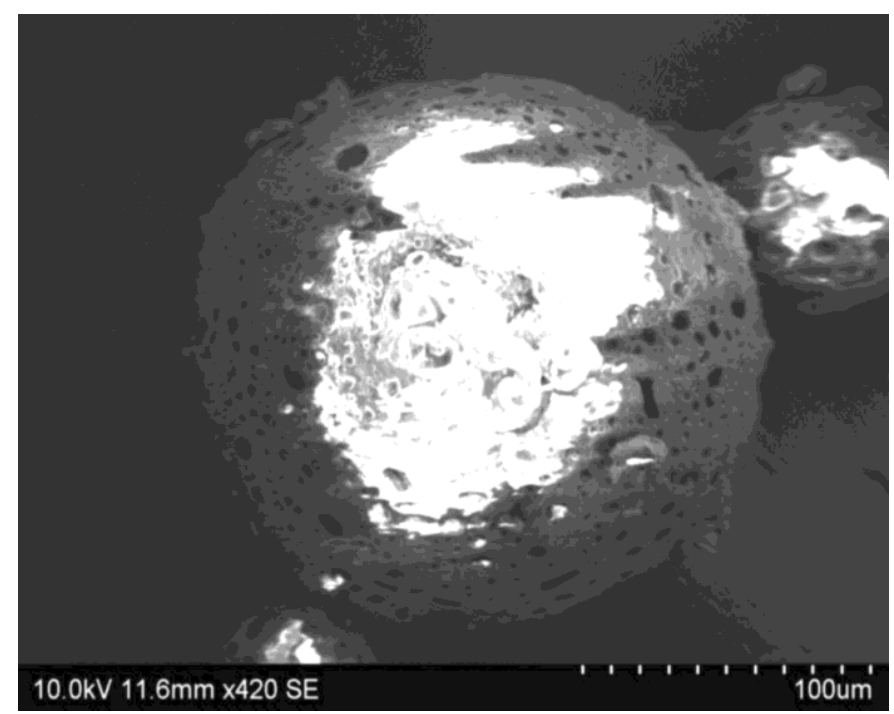

Fig. 4: SEM photomicrographs showing porous nature and spherical shape of cholestyramine based microspheres.

\section{Percentage buoyancy and drug entrapment:}

In Vitro Buoyancy percentage of the microspheres calcium silicate based Eletriptan hydrobromide was in the range of $76.15 \pm 0.55 \%$ to $93.80 \pm 1.75 \%$ at the end of $12 \mathrm{~h}$ above $88 \%$ ) and that of cholestyramine based Eletriptan Hydrobromide $77.54 \pm 1.73$ to $91.83 \pm 1.74 \%$ at the end of $12 \mathrm{~h}$ as shown in Table. 2. Good in vitro floating behavior was observed for all the microsphere formulation. This may be attributed to the low density calcium silicate within the system.

The percent drug entrapment of calcium silicate based Eletriptan hydrobromide in all the formulations was in the range of $53.02 \pm 4.97 \%$ to $75.29 \pm 1.93 \%$ found to be good at all levels of drug loading and that of cholestyramine based microspheres was in the range of $19.96 \pm 1.73 \%$ to $56.91 \pm 4.49 \%$ as in Table. 3 . The extent of loading influenced the particle size distribution of microspheres. When the loading was high, the proportion of larger particles formed was also high. With $75 \%$ 
entrapment, most of the particles(calcium silicate based) were in the size range of 84-100 micrometer, suitable for oral administration and with $57 \%$ entrapment, most of the particles(cholestyramine based) were in the size range of 58.5-68.9micrometer, As the concentration of total amount of polymer was increased, drug entrapment efficiency (drug content) was also increased. Formulation CS2B showed good $\%$ drug entrapment $(73.10 \pm 1.93 \%)$.

Table 2: In-vitro buoyancy studies of Calcium silicate and Cholestyramine based Eletriptan Hydrobromide floating microspheres.

\begin{tabular}{|c|c|c|}
\hline Formulation & $\begin{array}{c}\text { Total floating } \\
\text { time(hr) }\end{array}$ & \% of Buoyancy \\
\hline CS1A & $>24$ & $76.15 \pm 0.55$ \\
\hline CS1B & $>24$ & $86.81 \pm 0.96$ \\
\hline CS1C & $>24$ & $89.43 \pm 4.43$ \\
\hline CS2A & $>24$ & $86.21 \pm 1.12$ \\
\hline CS2B & $>24$ & $93.80 \pm 1.75$ \\
\hline CS2C & $>24$ & $90.96 \pm 1.21$ \\
\hline CH1A & $>24$ & $77.54 \pm 1.73$ \\
\hline CH1B & $>24$ & $80.49 \pm 1.57$ \\
\hline CH1C & $>24$ & $86.12 \pm 0.97$ \\
\hline CH2A & $>24$ & $82.77 \pm 0.48$ \\
\hline CH2B & $>24$ & $85.82 \pm 1.20$ \\
\hline CH2C & $>24$ & $91.83 \pm 1.74$ \\
\hline
\end{tabular}

Table 3: Drug entrapment of floating microspheres

\begin{tabular}{|c|c|}
\hline Formulation code & \% Drug entrapment \\
\hline CS1A & $53.02 \pm 4.97$ \\
\hline CS1B & $40.10 \pm 9.62$ \\
\hline CS1C & $61.87 \pm 0.57$ \\
\hline CS2A & $68.08 \pm 0.98$ \\
\hline CS2B & $75.29 \pm 1.93$ \\
\hline CS2C & $70.98 \pm 0.59$ \\
\hline CH1A & $19.96 \pm 1.73$ \\
\hline CH1B & $21.27 \pm 0.63$ \\
\hline CH1C & $24.08 \pm 0.45$ \\
\hline CH2A & $42.40 \pm 1.77$ \\
\hline CH2B & $46.99 \pm 0.79$ \\
\hline CH2C & $56.91 \pm 4.49$ \\
\hline
\end{tabular}

\subsection{Production yield:}

The percentage yield of floating microspheres of formulations (CS1A to CS2C) was found to be in the range of 94.64 to $98.37 \%$ and 96.936 to $98.86 \%$ in cholestyramine based formulations ( $\mathrm{CH} 1 \mathrm{~A}$ to $\mathrm{CH} 2 \mathrm{C}$ )

\subsection{In- vivo floating behavior:}

The in vivo floating behavior of formulation (CS2B and $\mathrm{CH} 2 \mathrm{C}$ ) hollow microspheres loaded with barium sulphate was investigated by radiographic images (X-ray photographs) of rabbit's stomach at specific periods. The amount of X-ray opaque material in these hollow was sufficient to ensure visibility by X-ray but at same time the amount of bariumsulphate $(100 \mathrm{mg}$ ) was low enough to enable the hollow microspheres to float. The hollow microspheres did not adhere to the gastric mucous and floating on the gastric fluid for about more the $12 \mathrm{~h}$. This formulation $\mathrm{CH} 2 \mathrm{C}$ hollow microsphere loaded with barium sulphate at $1^{\text {st }}$ hour. was evident by the $\mathrm{X}$-ray photographs taken at $1 \mathrm{~h}, \& 9 \mathrm{~h}$. It is shown in Fig. 5, Fig. 6, Fig.7 and Fig.8.

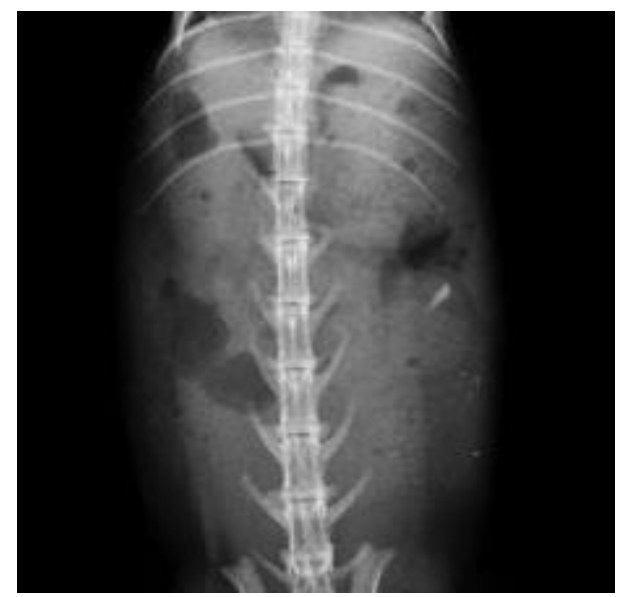

Fig 5: Radiographic images (X-ray photographs) of rabbit's stomach to investigate in vivo floating behavior of formulation CS2B hollow microspheres loaded with barium sulphate at $1^{\text {st }}$ hour.

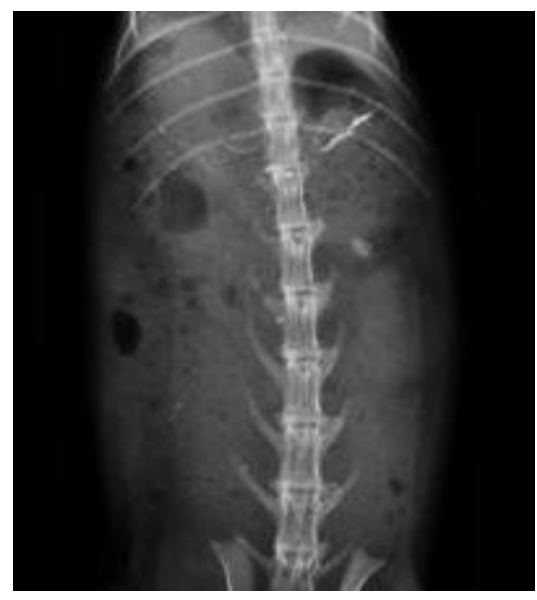

Fig 6: Radiographic images (X-ray photographs) of rabbit's stomach to investigate in vivo floating behavior of formulation CS2B hollow microspheres loaded with barium sulphate at $9^{\text {th }}$ hour.

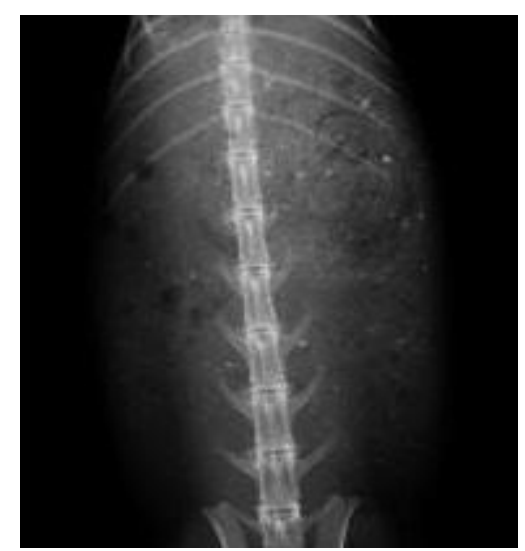

Fig 7: Radiographic images (X-ray photographs) of rabbit's stomach to investigate in vivo floating behavior of 


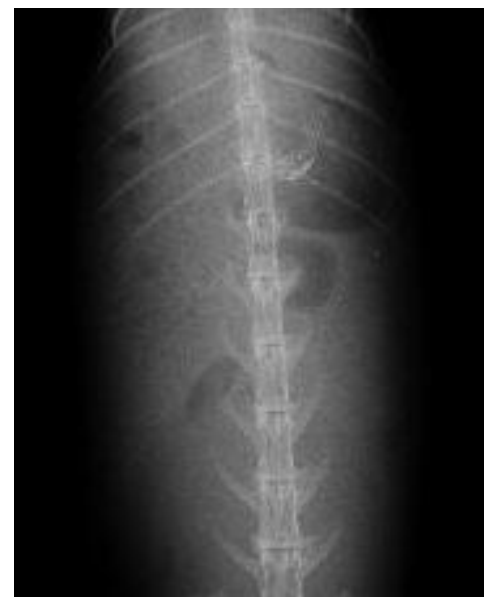

Fig. 8: Radiographic images (X-ray photographs) of rabbit's stomach to investigate in vivo floating behavior of formulation $\mathrm{CH} 2 \mathrm{C}$ hollow microspheres loaded with barium sulphate at $9^{\text {th }}$ hour.
Release of Eletriptan Hydrobromide from calcium silicate and cholestyramine based microspheres was evaluated in pH 2.0 as shown in Table. 4 and Table. 5. Calcium silicate based floating drug delivery system provides the possibility of enhancing the bioavailability and control the release of Eletriptan Hydrobromide exhibiting absorption window by prolonging the gastric emptying time of the dosage form, ensuring availability of drug at the absorption site for the desired period of time. The calcium silicate microsphere with adsorbed drug and polymer coating showed a drug release ranging from $92.08 \pm 3.59$ to 98.05 \pm 1.99 in Fig.9, when compared to cholestyramine based floating microspheres with drug release ranging from $92.60 \pm 3.92$ to $98.28 \pm 1.60$ as in Fig.10.The release of optimized formulations $\mathrm{CS} 2 \mathrm{~B}$ and $\mathrm{CH} 2 \mathrm{C}$ is depicted in Fig.11. From the mathematical modeling of dissolution profiles, as shown in Table. 6, CS2B showed higher regression co efficient 0.9898 following first order nonfickian diffusion kinetic model.

\section{In- vitro drug release study:}

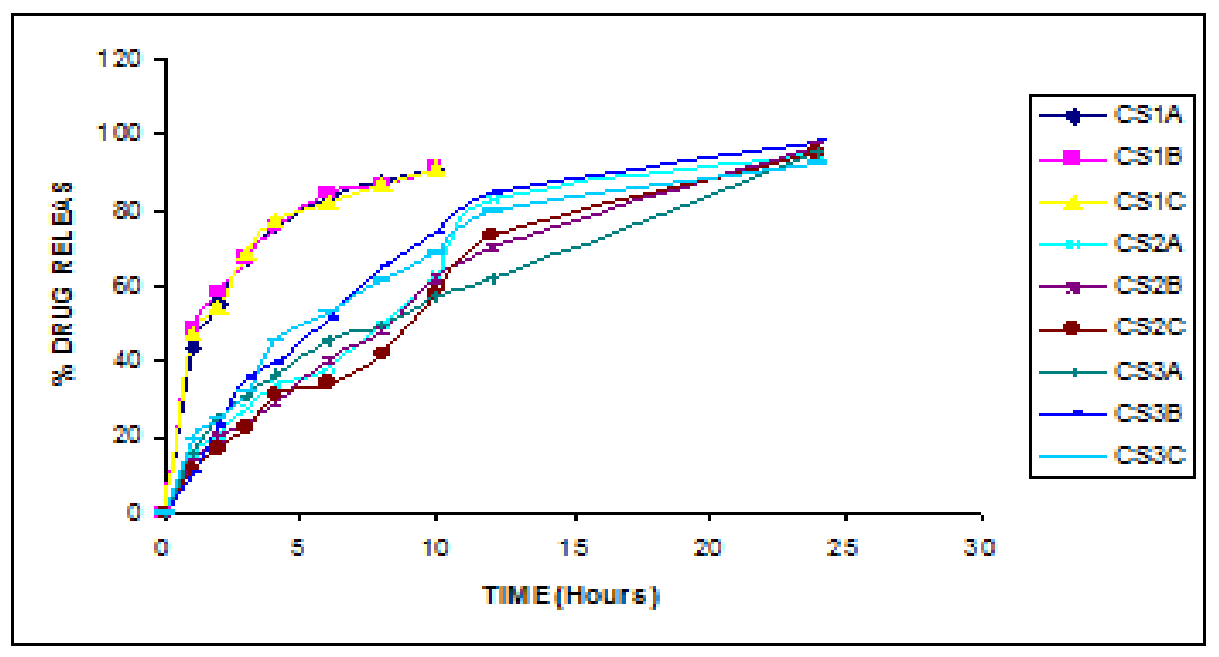

Fig 9: Cumulative percentage drug release in all Calcium silicate based microspheres

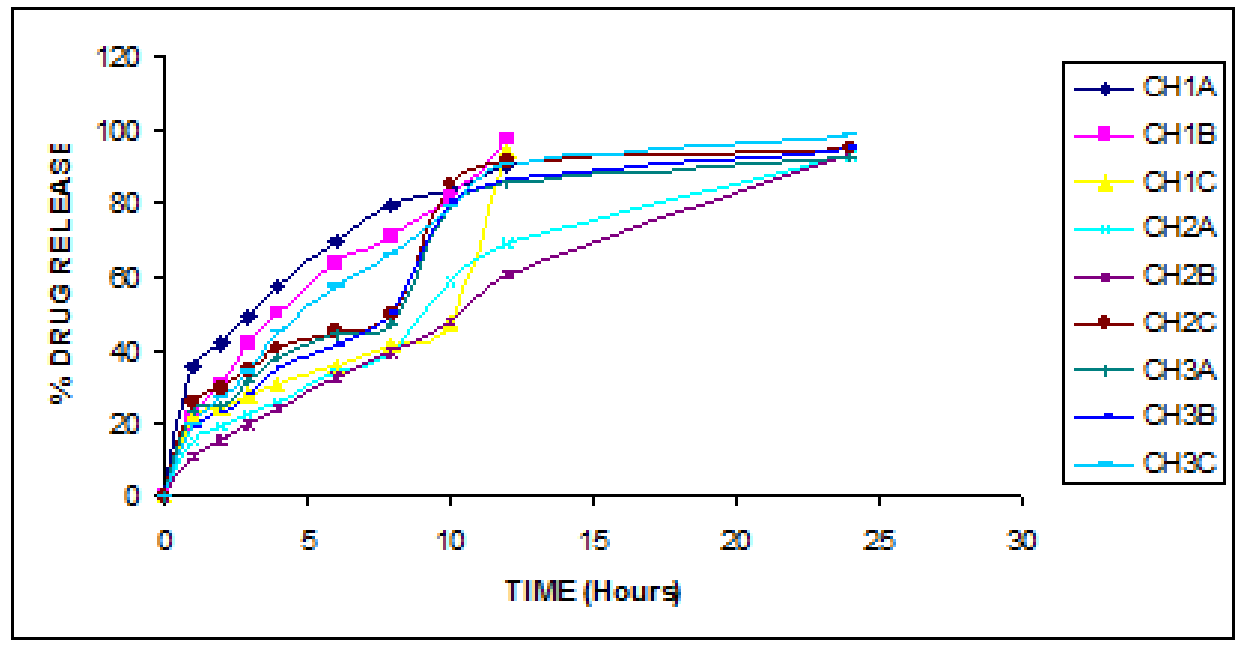

Fig 10: Cumulative percentage drug release in all Cholestyramine based microspheres. 


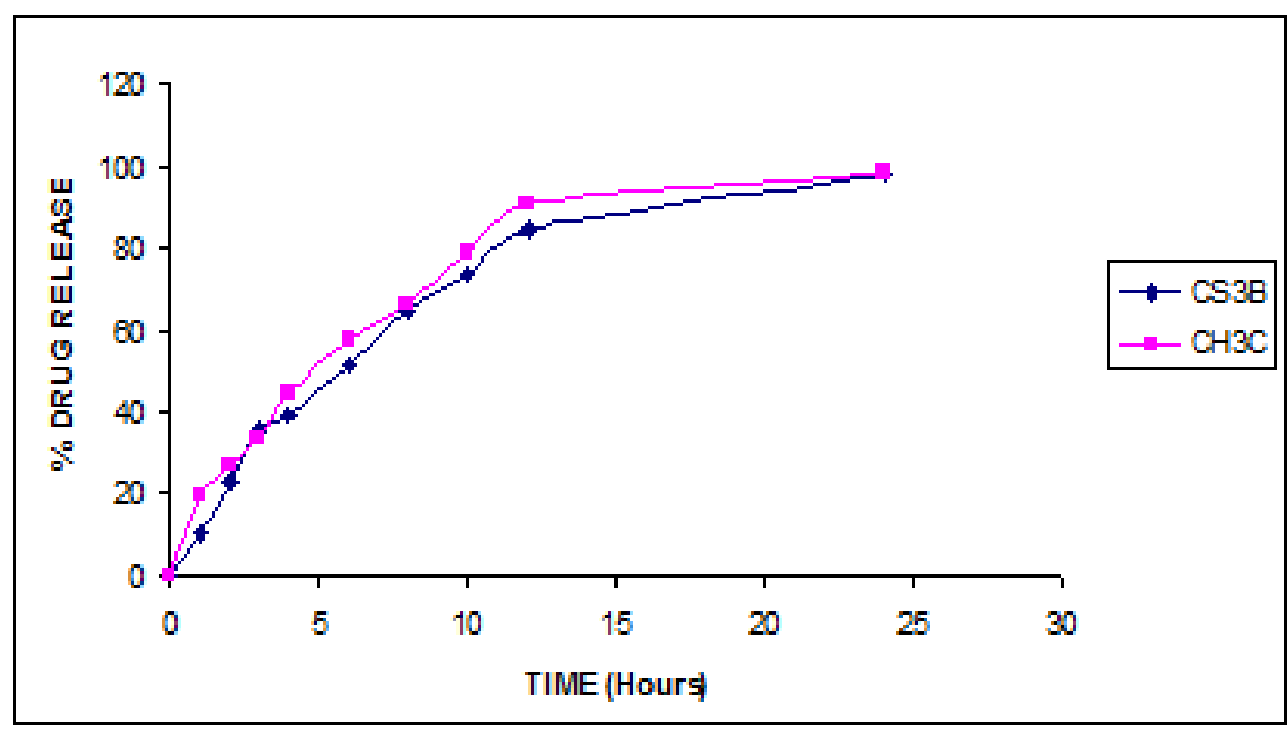

Fig 11: Cumulative percentage drug release in optimized formulations.

Table 4: Percentage drug release of Calcium silicate based Eletriptan Hydrobromide microspheres

\begin{tabular}{|c|c|c|c|c|c|c|}
\hline $\begin{array}{c}\text { Time } \\
\text { (Hours) }\end{array}$ & CS1A & CS1B & CS1C & CS2A & CS2B & CS2C \\
\hline 0 & $0.00 \pm 0.00$ & $0.00 \pm 0.00$ & $0.00 \pm 0.00$ & $0.00 \pm 0.00$ & $0.00 \pm 0.00$ & $0.00 \pm 0.00$ \\
\hline 1 & $15.96 \pm 4.33$ & $13.73 \pm 2.17$ & $12.06 \pm 0.09$ & $16.19 \pm 3.58$ & $10.69 \pm 1.02$ & $19.47 \pm 2.55$ \\
\hline 2 & $20.80 \pm 7.51$ & $19.81 \pm 2.18$ & $17.25 \pm 2.16$ & $25.41 \pm 0.77$ & $23.25 \pm 1.36$ & $25.08 \pm 4.14$ \\
\hline 3 & $27.92 \pm 9.49$ & $23.79 \pm 4.36$ & $22.47 \pm 2.16$ & $30.67 \pm 5.87$ & $35.37 \pm 1.54$ & $31.97 \pm 5.00$ \\
\hline 4 & $33.82 \pm 11.35$ & $29.55 \pm 4.39$ & $31.22 \pm 2.15$ & $36.59 \pm 4.57$ & $39.32 \pm 1.59$ & $45.77 \pm 3.39$ \\
\hline 6 & $38.50 \pm 11.41$ & $39.96 \pm 6.57$ & $34.14 \pm 4.32$ & $45.54 \pm 4.56$ & $51.16 \pm 1.70$ & $53.39 \pm 4.72$ \\
\hline 8 & $49.84 \pm 8.01$ & $48.68 \pm 4.45$ & $41.83 \pm 6.78$ & $49.91 \pm 5.93$ & $64.82 \pm 1.81$ & $61.31 \pm 3.19$ \\
\hline 10 & $63.24 \pm 8.04$ & $62.07 \pm 5.75$ & $57.68 \pm 8.34$ & $57.31 \pm 2.24$ & $73.67 \pm 1.86$ & $68.77 \pm 4.20$ \\
\hline 12 & $82.58 \pm 2.20$ & $70.28 \pm 5.78$ & $73.12 \pm 4.22$ & $62.25 \pm 0.09$ & $84.34 \pm 1.92$ & $79.77 \pm 6.26$ \\
\hline 24 & $95.28 \pm 6.02$ & $96.91 \pm 2.12$ & $95.40 \pm 4.65$ & $95.34 \pm 7.51$ & $98.05 \pm 1.99$ & $92.08 \pm 3.59$ \\
\hline
\end{tabular}

Table 5: Percentage drug release of Cholestyramine based Eletriptan Hydrobromide microspheres

\begin{tabular}{|c|c|c|c|c|c|c|}
\hline $\begin{array}{c}\text { Time } \\
\text { (Hours) }\end{array}$ & CH1A & CH1B & CH1C & CH2A & CH2B & CH2C \\
\hline 0 & $0.00 \pm 0.00$ & $0.00 \pm 0.00$ & $0.00 \pm 0.00$ & $0.00 \pm 0.00$ & $0.00 \pm 0.00$ & $0.00 \pm 0.00$ \\
\hline 1 & $15.49 \pm 8.73$ & $11.19 \pm 2.18$ & $25.81 \pm 5.34$ & $23.31 \pm 4.17$ & $19.24 \pm 5.48$ & $19.75 \pm 5.71$ \\
\hline 2 & $19.20 \pm 6.40$ & $15.50 \pm 0.03$ & $29.58 \pm 4.65$ & $24.31 \pm 4.29$ & $22.47 \pm 4.03$ & $27.11 \pm 5.13$ \\
\hline 3 & $21.93 \pm 5.79$ & $19.84 \pm 2.17$ & $33.99 \pm 7.48$ & $30.95 \pm 9.85$ & $27.59 \pm 5.88$ & $33.38 \pm 10.13$ \\
\hline 4 & $25.43 \pm 7.12$ & $23.82 \pm 4.34$ & $40.30 \pm 11.04$ & $37.87 \pm 11.59$ & $34.74 \pm 1.80$ & $44.44 \pm 3.71$ \\
\hline 6 & $33.69 \pm 6.94$ & $32.20 \pm 4.37$ & $44.65 \pm 11.72$ & $44.07 \pm 11.81$ & $41.31 \pm 1.95$ & $57.43 \pm 9.06$ \\
\hline 8 & $39.37 \pm 8.06$ & $39.01 \pm 7.42$ & $49.39 \pm 12.66$ & $47.44 \pm 11.93$ & $50.41 \pm 3.23$ & $65.87 \pm 9.81$ \\
\hline 10 & $58.46 \pm 2.31$ & $47.47 \pm 2.27$ & $84.91 \pm 0.80$ & $79.45 \pm 3.20$ & $79.93 \pm 6.31$ & $78.98 \pm 5.40$ \\
\hline 12 & $69.41 \pm 5.15$ & $60.73 \pm 0.12$ & $91.37 \pm 1.13$ & $85.63 \pm 1.81$ & $86.62 \pm 5.91$ & $90.41 \pm 2.92$ \\
\hline 24 & $93.16 \pm 2.49$ & $93.43 \pm 2.96$ & $94.74 \pm 1.53$ & $92.60 \pm 3.92$ & $94.84 \pm 0.95$ & $98.28 \pm 1.60$ \\
\hline
\end{tabular}


Table 6: Regression coefficient $\left(\mathbf{R}^{2}\right)$ values of Eletriptan Hydrobromide floating microspheres for different kinetic models

\begin{tabular}{|c|c|c|c|c|c|}
\hline \multirow{2}{*}{ Formulation } & Zero-order & First-order & \multicolumn{2}{c|}{ Higuchi } & \multicolumn{2}{c|}{ Korsmeyer-Peppas } \\
\cline { 2 - 6 } & \multicolumn{2}{|c|}{$\mathrm{R}$} & 0.9645 & 0.7262 & 0.2937 \\
\hline CS1A & 0.7697 & 0.963 & 0.9492 & 0.0016 & 0.0518 \\
CS1B & 0.7435 & 0.9513 & 0.9336 & 0.7121 & 0.3271 \\
CS1C & 0.7508 & 0.9525 & 0.9617 & 0.5794 & 0.5043 \\
CS2A & 0.8963 & 0.9676 & 0.9786 & 0.5985 & 0.4657 \\
CS2B & 0.9415 & 0.9506 & 0.9641 & 0.637 & 0.5819 \\
CS2C & 0.9381 & 0.9589 & 0.9961 & 0.65 & 0.5477 \\
CS3A & 0.918 & 0.948 & $\mathbf{0 . 9 6 7 1}$ & $\mathbf{0 . 6 9 2 7}$ & $\mathbf{0 . 6 2 8}$ \\
CS3B & $\mathbf{0 . 8 4 2 6}$ & $\mathbf{0 . 9 8 9 8}$ & 0.9706 & 0.6829 & 0.523 \\
CSC & 0.8589 & 0.9858 & 0.9859 & 0.6721 & 0.3118 \\
CH1A & 0.8853 & 0.9668 & 0.9916 & 0.6877 & 0.522 \\
CH1B & 0.9593 & 0.8651 & 0.7873 & 0.4446 & 0.3557 \\
CH1C & 0.8414 & 0.6018 & 0.9509 & 0.5421 & 0.4125 \\
CH2A & 0.9444 & 0.9629 & 0.962 & 0.591 & 0.5242 \\
CH2B & 0.9793 & 0.9389 & 0.639 & 0.5982 & 0.3578 \\
CH2C & 0.8016 & 0.8804 & 0.921 & 0.5708 & 0.3507 \\
CH3A & 0.8285 & 0.9195 & 0.9202 & 0.6349 & 0.4684 \\
CH3B & 0.8447 & 0.9446 & 0.9592 & 0.6772 & 0.4734 \\
CH3C & 0.8187 & 0.9847 & & & \\
\end{tabular}

Table 7: Stability study for drug content of prepared CS2B and CH2C microspheres

\begin{tabular}{|c|c|c|c|}
\hline Sampling (days) & Drug content (\%) CS2B & Drug content (\%) CH2C & Stability condition \\
\hline 0 & $75.29 \pm 1.93$ & $56.91 \pm 4.49$ & \multirow{6}{*}{$40{ }^{\circ} \mathrm{C} / 75 \% \mathrm{RH}$} \\
\hline 7 & $75.25 \pm 1.24$ & $56.75 \pm 3.98$ & \\
\hline 15 & $75.21 \pm 1.87$ & $56.63 \pm 1.55$ & \\
\hline 30 & $75.18 \pm 1.85$ & $56.56 \pm 1.98$ & \\
\hline 60 & $75.16 \pm 1.72$ & $56.35 \pm 2.11$ & \\
\hline 90 & $75.11 \pm 1.22$ & $56.14 \pm 1.65$ & \\
\hline
\end{tabular}

\subsection{Accelerated Stability studies:}

The objective of stability studies is to predict the shelf life of a product by accelerating the rate of decomposition, preferably by increasing the temperature and $\mathrm{RH}$. The RSM containing selected formulation $\mathrm{CS} 2 \mathrm{~B}$ and $\mathrm{CH} 2 \mathrm{C}$ was subjected to stability studies carried out by storing at $40^{\circ} \mathrm{C} / 75 \% \mathrm{RH}$ for 3 months (climatic zone IV condition for accelerated testing) to assess their stability. These samples were analyzed and checked for changes in physical appearance and drug content at regular intervals. The obtained data is presented in Table 7. From the table, it is clear that the formulation did not undergo any chemical changes/interaction during the study period.

\section{CONCLUSION:}

The present formulation study of Eletriptan Hydrobromide was performed in an attempt to prepare floating drug delivery system consisting of floating multiple unit system. Incorporation of Calcium silicate in the microspheres proved to be an effective method to achieve the desired release behavior and buoyancy than incorporation of cholestyramine. The performance of these formulations was evaluated and the effect of various formulation variables was studied. The designed system, combining excellent buoyant ability and suitable drug release pattern, could possibly be advantageous in terms of increased bioavailability of Eletriptan Hydrobromide. Major advantages of the system include: (i) ease of preparation, (ii) good buoyancy, (iii) high encapsulation efficiency, and (iv) sustained drug release over several hours.

\section{ACKNOWLEDGEMENT:}

The authors wish to thank Finosa Pharmaceuticals, Hyderabad for providing the gift sample of Eletriptan Hydrobromide. The authors are thankful to D.Manohar Reddy, chairman, Trinity College of Pharmaceutical Sciences for their kind help and providing all necessary facilities.

\section{CONFLICTS OF INTEREST:}

The authors declare that they have no conflicts of interest. 


\section{REFERENCES}

1. Najmuddin.M, Aejaz Ahmed, Sachin Shelar, Patel.V, Khan.T. Floating Microspheres of Ketoprofen, Formulation and Evaluation. Int J of Pharm and Pharm Sci. 2010:164-168.

2. Baumgastners, Kristal.J, Vreer.F, Vodopivec.P, Zorko.B. Optimization of floating matrix tablet and evaluation of there gastric residence time. Int J Pharm. 2000: 95: 125 - 130.

3. Garg.R, Gupta.G.d. Progress in controlled gastro retentive drug delivery systems. Trop J of Pharm Res. 2008: 7(3) 1055-1066.

4. Singh.Y.T, Singh.P.N, Ojha.G.R. Development and evaluation of floating microspheres of Verpamil hydrochloride. Braz $\mathrm{J}$ of Pharm Sci. 2007: 43 (4): 529 - 534.

5. Sam.M.T, Devi S.G, Prasanath.V.V, Vinod.B. NSAIDs as microspheres. The Int J of Pharmacology. 2008: 6 (1): 27 - 38.

6. Tanwar.Y.S, Floating microspheres, Development, characterization and application. Pharmaceutical Reviews. 2006: 4 (3). (Available at www.pharmainfo.net).

7. Kumara Swamy.G, Kumar.J.M.R, Sheshagiri Rao.J.V.L.N, Ashok Kumar.U, Vinaya Snehalatha.E. Spectrophotometric Method for the Estimation of Eletriptan Hydrobromide in Pure and Tablet Dosage Forms. Int $\mathrm{J}$ of Chem and Analytical Science. 2011: 2(8):123-125.

8. Jain.S.K, Awasthi.A.M, Jain.N.K, Agrawal.G.P. Calcium silicate based microspheres of repaglinide for gastroretentive floating drug delivery, Preparation and in vitro characterization. J of Cont Rel. 2005: 107 300-309.

9. Jelvehgari.M, Nokhodchi.A, Rezapour.M, Valizadeh.H. Effect of Formulation and Processing Variables on the Characteristics of Tolmetin Microspheres Prepared by Double Emulsion Solvent Diffusion Method. Indian J Pharm Sci. 2010: 72(1) : $72-78$.
10. Ashwini.R.M, Mangesh.R.B, Rahul.R.P, Nilkanth.S.P, Devaki.C.U. Formulation and Optimization of Drug-Resin Complex Loaded Mucoadhesive Chitosan Beads of Repaglinide Using Factorial Design. American $\mathrm{J}$ of Medicine and Medical Sci. 2012: 2(4): 62-70.

11. Martin, Physical Pharmacy, IVth ed., Lea Febiger, Philadelphia.1993. 431- 432.

12. Ritschel.W.A, Thompson.G.A, Lucker.P.W, Wetzelsberger.K. Biopharmaceutics evaluation of etofylline clofibrate and its drug formulation, Arzneim.-Forsch/ Drug Res.1980:30: 20202023.

13. Kilicarslan.M, Baykara.T. The effect of the drug/polymer ratio on the properties of Verapamil $\mathrm{HCl}$ loaded microspheres. Int $\mathbf{J}$ Pharm.2003:252: 99-109.

14. Srivastava.A.k, Ridhurkar.D.N, Wadhwa.S. Floating microspheres of cimetidine: formulation, characterization and in vitro evaluation. Acta Pharm. 2005:55: 277-285.

15. Joseph.N.J , Lakshmi.S, Jayakrishnan.A, Acta Pharm. 2005:55: 277-285.

16. Gangadharappa.H.V, Biswas.S. Getyala.A, Vishal Gupta.N, Pramod Kumar.T.M Development, In vitro and In vivo Evaluation of Novel Floating Hollow Microspheres of Rosiglitazone Maleate. Der Pharmacia Lettre. 2011: 3 (4):299316.

17. Rao.K.R, Senapati.P, Das.M.K. Formulation and in vitro evaluation of ethyl cellulose microspheres containing zidovudine. J Microencapsul.2005:22:863-876.

18. Mingli.Y, Sungwon Kim, Kinam Park. Issues in long-term protein delivery using biodegradable microparticles. J of Cont Rel. 2010: 146:241-260.

\section{Figure legends}

